\title{
Prix Fermat 1991
}

Annales de la faculté des sciences de Toulouse $6^{e}$ série, tome $1, \mathrm{n}^{\circ} 3$ (1992), p. 293-294

<http://www.numdam.org/item?id=AFST_1992_6_1_3_293_0>

(C) Université Paul Sabatier, 1992, tous droits réservés.

L'accès aux archives de la revue «Annales de la faculté des sciences de Toulouse » (http://picard.ups-tlse.fr/ annales/) implique l'accord avec les conditions générales d'utilisation (http://www.numdam.org/conditions). Toute utilisation commerciale ou impression systématique est constitutive d'une infraction pénale. Toute copie ou impression de ce fichier doit contenir la présente mention de copyright.

\section{NumDam}

Article numérisé dans le cadre du programme

Numérisation de documents anciens mathématiques

http://www.numdam.org/ 


\section{Prix Fermat 1991}

Organisé par l'Université Paul Sabatier de Toulouse et Matra Marconi SPACE, le prix Fermat de recherches en mathématiques 1991 a été décerné à JEAN-LOUIS COLLIOT-THÉLÈnE pour ses travaux sur l'arithmétique des variétés rationnelles, recherche dont une grande partie a été menée conjointement avec JEAN-JACQUES SANSUC.

La citation du jury ainsi qu'un article de J.-L. Colliot-ThÉLÈnE basé sur son exposé à l'occasion de la remise du prix à Toulouse paraissent dans ce numéro des Annales de la Faculté des Sciences de Toulouse.

" La recherche de critères permettant de décider si une équation diophantienne a des solutions rationnelles ou non est un problème difficile. Si on ne se limite pas à des classes particulières d'équations, on sait maintenant qu'il n'y a pas d'algorithme (solution négative au dixième problème de Hilbert).

Pour qu'une équation donnée à coefficients rationnels admette des solutions rationnelles, il est évidemment nécessaire qu'elle en admette dans le corps des nombres réels; plus précisément il faut aussi qu'elle en admette dans tous les complétés du corps des rationnels. Ces conditions sont faciles à tester. Pour une forme quadratique (polynôme homogène de degré 2), cette condition nécessaire est aussi suffisante; on dit alors que le principe de Hasse est valable. Dès le degré 3 , le principe de Hasse n'est plus valable. Un exemple de Chatelet, repris par Manin, a fourni le point de départ des travaux de Colliot-Thélène et Sansuc, qui ont mis en évidence une obstruction à ce principe de Hasse, et qui montrent que c'est la seule obstruction pour certaines familles de variétés. Cela fournit des algorithmes pour déterminer si certaines équations ont ou non des solutions rationnelles.

Les résultats obtenus par Sansuc et Colliot-Thélène sont dans la ligne directe de certains travaux de Fermat. Ainsi ils ont établi une condition nécessaire et suffisante pour qu'un entier positif soit somme de deux carrés et d'une puissance quatrième dans le corps des rationnels (Colliot-Thélène et Sansuc, Crelle 374 p. 93) :

écrivons $n$ sous la forme $2^{4 r+s} m$, avec $m$ impair $r$ et $s$ positifs et $s$ entre 0 et 3 . Alors $n$ est somme de deux carrés et d'une puissance quatrième, sauf si :

- soit $s=0$ et $m$ congru a 7 modulo 8 ;

- soit $s=2$ et $m$ congru a 3 modulo 4 .

La première exception est claire, c'est la condition de Gauss : dans ce cas, $n$ n'est pas somme de trois carrés. Le plus frappant dans cet énoncé est que la condition 2-adique soit une condition suffisante. On ne sait rien dire sur la représentation de $n$ sous la forme $x^{2}+y^{2}+z^{4}$ avec $x, y, z$ entiers.

Les obstructions de nature réelle sont essentiellement les seules pour l'existence d'une solution rationnelle simultanée pour un système de deux formes quadratiques en au moins 
9 variables. C'est l'analogue du résultat de Meyer pour une forme quadratique a au moins 5 variables. La méthode du cercle (Heath-Brown) n'a donné jusqu'à présent que le cas d'au moins 10 variables, en supposant de surcroît le système de deux formes non singulier.

Dans un article en commum avec Kanevsky et Sansuc, article référencé ci-après par $\mathrm{CT} / \mathrm{K} / \mathrm{S}$, Colliot-Thélène étudie les surfaces cubiques diagonales

$$
a x^{3}+b y^{3}+c z^{3}+d t^{3}=0 .
$$

À l'heure actuelle, on n'a pas d'algorithme pour décider si une telle équation a des solutions rationnelles; les conditions de congruence ne sont pas suffisantes (Cassels/Guy 1966). À l'époque du contre-exemple de Cassels-Guy, l'ordinateur avait produit quelques 200 équations a petits coefficients entiers, avec des solutions modulo tous les entiers, et sans petite solution rationnelle. Cassels réussit a montrer qu'une de ces équations n'avait pas de solution rationnelle. À l'époque, personne n'avait idée comment traiter une seule des autres équations. Dix ans plus tard, Bremner (1978) réussit a traiter une autre équation dans la liste (voir l'exposé de Colliot-Thélène au Séminaire de Théorie des nombres de Paris en 1984-1985 pour l'histoire et la phéhistoire du problème). CT/K/S ont donné un algorithme systématique pour calculer l'obstruction de Brauer-Manin au principe de Hasse pour de telles surfaces sur les rationnels; ils utilisent la cohomologie des groupes bicycliques, les théorèmes de dualité de Lichtenbaum pour les courbes elliptiques sur les corps p-adiques, ainsi que les formes explicites pour le corps de classes.

Voici un exemple de résultat qu'ils obtiennent :

s'il existe un nombre premier qui divise un coefficient et un seul, alors il n'y a pas d'obstruction de Brauer-Manin.

De fait, les coefficients de Cassels-Guy étaient $(5,9,10,12)$ et ceux de Bremner $(1,4$, 10, 25).

L'ordinateur étudia ensuite toutes les équations avec $a, b, c, d$ entiers plus petits que 100. Dans ce domaine, pour toutes les équations survivant a l'élimination par les congruences puis a l'élimination par l'obstruction de Brauer-Manin calculée par l'algorithme de $\mathrm{CT} / \mathrm{K} / \mathrm{S}$, il trouva des solutions entières (de hauteur plus petite que 8000 ). Au passage, cela régla la situation pour la "petite" liste d'équations de CasselsGuy.

Cela donne quelque poids à la conjecture que l'obstruction de Brauer-Manin est ici la seule, et que l'algorithme cité permet effectivement de décider si une surface cubique diagonale a un point rationnel...

Une autre contribution importante de Sansuc et Colliot-Thélène, en collaboration avec Beauville et $S$ winnerton-Dyer, est la réponse négative à un vieux problème de Zariski : ils donnent un exemple d'une extension $K$ du corps $\mathbb{C}$ des nombres complexes, qui n'est pas de la forme $\mathbf{C}\left(X_{1}, \ldots, X_{n}\right)$ avec des $X_{i}$ algébriquement indépendants, mais qui est telle que le corps des fractions rationnelles $K(T)$ soit de cette forme. En termes géométriques, cela signifie qu'ils construisent une variété algébrique sur $\mathbb{C}$ qui n'est pas rationnelle, mais qui l'est stablement (c'est-à-dire après multiplication par une droite).

Colliot-Thélène et Sansuc sont devenus les chefs de file d'une véritable école consacrée à l'arithmétique des variétés rationnelles, qui met en œuvre une grande variété de moyens : géométrie algébrique, $K$-théorie, formes quadratiques, méthode du cercle, etc.

L'idée qui est au cœur de leurs travaux est celle qui était déjà au cœur des travaux de Fermat." 